\title{
The Epidemiology and Prevention of Leprosy.
}

R. G. Cochrane.

(Reprinted from "International Journal of Leprosy," October-December, 1934.)

INTRODUCTION.

7 HERE are still many aspects of leprosy which have yet to be elucidated, but we can to-day take a bird'seye view of the problem in a way which was impossible ten or fifteen years ago. There has evolved a new outlook, the chief result of which is the increasing number of cases with early or slight lesions which are being seen. This has complicated the clinical picture, and as will be shown has a most important bearing on its public health aspects. In this connection I propose to put forward a hypothesis which has arisen as a result of experience gained in studying the disease in many countries and under many varying conditions, and which may help to simplify the problem of controlling and ultimately eliminating it.

A basic consideration is that while leprosy is doubtless mildly contagious it seems to be no more than just pathogenic to man ; that in order for it to gain a sufficient footing in the body to cause damage a considerable disturbance of normal resistance is necessary. The disease is closely analogous to tuberculosis in many respects; but the causative organism is peculiar in that, though it is a parasite, it seems in many instances to establish an almost perfect commensalism with the tissues. This is illustrated by the type of case, not infrequently seen, in which there is little clinical evidence of the infection, but on examination innumerable bacilli are found wherever a scraping is taken ; a balance seems to have been reached between the body and the bacillus whereby the latter lives and multiplies but causes little or no damage to the host. There seems to be little evidence of the production of toxins, and the reaction to the organisms by the body seems to be a reaction to a foreign body rather than to a toxin-producing organism.

AgE At INFECTION.

The hypothesis that leprosy is usually acquired in childhood or early adolescence is being more and more generally accepted. Lampe $(1,2)$ states that he is firmly of that belief, and holds that active measures should be concentrated chiefly on the children. It is also believed by many that this period is one in which, if the infection is 
implanted, it is most liable to spread and set up active disease. It seems entirely probable that a large proportion of cases do become infected during these periods, and that once adult life is reached the chances of acquiring the disease are greatly diminished. However, there is reason to believe that there are many cases in which infection is actually established but does not go on to the active disease. If a patient has had only slight evidence of a leprotic infection in childhood, and if this has remained inactive during the stress and strain of adolescence, the chances of it becoming active are slight. There is, of course, the possibility that latent foci may light up even under the most unexpected conditions, but preventive measures on a large scale cannot be formulated upon the exceptions to what may be found to be a general rule.

On the basis of the foregoing, one of four things may happen if a child acquires leprosy :-

(1) The disease may advance into the more active stages and the individual become progressively worse.

(2) There may be a stationary or latent period and then, owing to lowered resistance, the disease may light up many years later.

(3) The infection may remain stationary and the lesion become naturally arrested, though remaining evident.

(4) The lesion may disappear entirely.

The ABortive CASe.

As a result of the increased interest shown in leprosy, and of the emphasis on surveys, cases of categories (3) and (4) - the abortive cases-are coming to light in both children and adults, whereas previously little or nothing was known about them. That such cases occur need not occasion surprise. One of the special features of leprosy is its selfhealing nature. Therefore, it seems quite as reasonable that leprosy should be naturally arrested in an early stage as that this may happen in late ones, as unquestionably is quite frequently the case. As a matter of fact there is every reason to believe that in many individuals who are in good health, and whose resistance is relatively high-though not quite high enough to prevent infection entirely-the disease does become arrested in the early stages.

There is an important parallel in tuberculosis, where many individuals have foci of the infection but show no clinical symptoms and do not pass into the more advanced stages-these, too, are " abortive cases." The chief difference between such cases in tuberculosis and leprosy is that in the former there is no means apart from a suspicious 
X-ray photograph whereby a patient with a slight early, latent or abortive lesion can be detected, whereas in leprosy a clinical diagnosis of a suspicious skin patch can often be made when there is no evidence of general activity of the disease, and such lesions can easily be observed for years.

I am aware that many believe that there is another class of abortive case in endemic areas, namely, persons who harbour the leprosy bacilli in deep foci and never show any external lesion. Such cases, if they exist, are more closely analogous to the undetected tuberculosis infections, but they do not come into the present discussion because they are never recognised.

Because of the existence of abortive cases, and of the numerous cases with extremely early or slight lesions that are being found in many places as a result of modern activities, entirely wrong conclusions are liable to be drawn from ordinary data on the incidence of the disease. If, for instance, a survey of an area has revealed a high incidence of leprosy it is only natural to assume that it is on the increase, and that all the cases which have been discovered need provision for active treatment. However, increasing knowledge of the early and especially the abortive cases indicates that our attitude may need modifying.

The Abortive Case and Treatment.

It seems very likely that indiscriminate treatment of every person found to have signs of leprotic infection may eventually bring modern treatment into disrepute. In treating all and sundry, especially in out-patient centres, one is treating among the adults those on the one hand who have abortive infections, and on the other hand those whose bodies are so invaded by the organism that there is little if any hope of bringing them to a stage of non-infectivity. Because of the lack of selection of cases in many out-patient centres favourable results are difficult to obtain within any reasonable period, and the whole work is adversely criticised.

With special reference to the children with inactive, very early or abortive lesions, one cannot agree with the view that it is wrong not to treat all cases regardless of type and activity of the lesion. We have no evidence that the present anti-leprosy drugs can prevent the development of further lesions in such cases. A recent article indicates that " drug treatment does not prevent these early developments of leprosy in young children," and that after the earliest lesions have appeared specific remedies do not prevent the development of more active forms of the disease (3). From this it would seem that proper living 
conditions constitute our main defence in such cases. Care of this nature probably gives the child an 80 per cent. chance of not developing further lesions; in many instances the suspected area will return to normal in a few years.

\section{The Abortive Case in Practice.}

It is most important to consider these things when a diagnosis of leprosy is made. The physician must ever remember the social factor, that a definite diagnosis often blasts the patient's life for ever. If the patient be a girl it may completely ruin her chances of marriage. It is also to be realised that it is probably as useless to give special remedies to the abortive cases as to the advanced secondary cases. In consideration of these facts, and of the existence of abortive cases among those found with very early or slight lesions, I believe that the general statement may be made that when such evidence of a leprotic infection is found it does not necessarily follow that either treatment or isolation should be instituted. It seems legitimate to conclude that, apart from examining such cases every six months, no further action need be taken unless and until there is evidence of activity of the disease.

\section{DESCRIPTION OF ABORTIVE Lesions.}

For these reasons, and others to be discussed, it is important that all leprosy workers should recognise the existence of the abortive type of case, and that the clinicians should be able to recognise it. Therefore it may be well at this point to endeavour to set down as carefully as possible the guiding points for the recognition of these lesions as seen in both adults and children. It may be said here that Mitsuda's test may be found of value in helping to decide whether a case is an abortive one or not, at least in adults.

Lesions in Adults. - In an adult it is a fairly simple matter to be reasonably sure when such lesions exist.

(1) A single skin lesion which has no sign of activity whatever, and has been in existence for some length of time -a year or more. If the history shows that the patch has been noticed only recently (within six months) it would be wise to keep the person under observation. If this cannot be done it may be necessary to treat the patient in order to insure his attendance for observation; an exception might be in the case of a healthy adult over 30 years of age, in which case isolated inactive patches of a suspicious nature may be left alone. It should be remembered that a supposedly early patch may be changed to scar tissue by intradermal injections, but in doing this it cannot legitimately 
be concluded that one is producing any effect on a leprotic infection unless some previous evidence of activity has been demonstrated.

(2) Multiple hypopigmented patches which have been in existence for a considerable time, with no other clinical sign such as anesthesia, enlarged nerves, etc. Such cases, while not coming under the heading of abortive cases, probably represent naturally arrested cases. The loss of pigment may be permanent and it may be a waste of time to endeavour to restore pigment to the affected areas.

(3) Neural lesions where one main nerve is thickened without tenderness, with no other clinical manifestation of the disease.

Lesions in Children.-Recognition of the abortive lesion in children is more difficult, but the following points may prove helpful :

(1) Unless they are absolutely typical, suspicious patches on the face may be disregarded except when accompanied by signs elsewhere. Hypopigmentation on the face is closely simulated by the results of septic infections, e.g., impetigo.

(2) If a child has only a single patch which does not show signs of activity, it is better to see that the child is placed under healthy surroundings than to treat it.

I wish to emphasise by repetition that with children diagnosis is more difficult than with adults, and that the responsibility of the physician is greater. I hold that whatever may be our views concerning the absolute efficacy of present treatment-a subject to be touched on again-we have no right to withhold the special remedies from any active case. On the other hand, as has been said, in treating as a leper a child who has a suspicious patch one is not certain of doing it any good with respect to the infection, and one is certain to do it harm socially. Therefore, unless there is definite evidence of activity of the disease in such cases there should be great hesitancy about applying the special remedies. In the event of a child being found to be an active case the situation is, of course, very different ; he should be put under active treatment. If the case proves to be an "open " infectious one he should, for the sake of other children if not for his own, be withdrawn from school and isolated.

\section{The Public Health Aspect.}

The position of the abortive case in general anti-leprosy measures is a matter of the greatest importance, one that should be given most careful consideration. As has been pointed out, this element may entirely vitiate conclusions 
drawn from mere numbers of persons found by survey to show evidence of leprotic infection. I was first impressed by this aspect of epidemiology during a tour in Africa a few years ago, when I was struck by the differences in the types of the disease seen in different places. In the Bahr-el-Ghazal Province of the Sudan especially I noted a large number of adults who had comparatively mild forms of the disease. This aspect of leprosy I have emphasised in a report to the Government of Ceylon on an investigation recently made there :-

"If this type is comparatively common, then it means that measures for prevention taken on information concerning the number of sufferers may be laying emphasis on entirely the wrong areas."

The question of what measures should be taken has not yet been satisfactorily settled. During the past few years schemes for the control of leprosy have emphasised chiefly the establishment of treatment centres and the treating of every case presented. However, a compilation made by the Mission to Lepers covering the past six years shows that in India the number of cases that are healed averages 9 per cent. of those treated. Some institutions have published figures as high as 17 per cent., but even in that case it means that a very large percentage do not become completely clear of the disease.

While I believe that we have made a great advance in treatment during the past ten years, it is nevertheless to be stressed that the present treatment methods do not necessarily benefit every type of case. Those which benefit most are those active cases in which the bacillus has not completely invaded the body; if statistics were confined to such cases the percentages of recoveries would be considerably higher than those indicated above. However, the facts as they exist strongly indicate that the stress which has been placed on out-patient treatment in a leprosy control campaign may need modifying. It may be noted that at the All-India Conference, held in Calcutta in March, 1933, the emphasis tended to swing from treatment to prevention (4). a Modified Public Health Plan.

There are still other factors that bear on the public health aspect of the problem. Leprosy is far more prevalent in some endemic centres than hitherto imagined, and in places the incidence that has been revealed by the work of the past ten years is such that unless the figures are interpreted properly they may be very discouraging. Leprosy, after all, is only one of the many endemic diseases in any 
country and the treating of even every early case would involve an expenditure of money and absorption of time out of all proportion to its importance. The problem which this disease presents is, as a whole, so difficult that it is humanly impossible to cope with it completely, from all aspects, in any country where the disease is very prevalent. A system adequate to that would engulf the medical resources of any budget. However, one may suggest that to attempt such a colossal task is perhaps unnecessary, but that a fresh viewpoint and a modified plan of campaign are called for.

In view of the foregoing, certain principles should be laid down in order that time may not be wasted, and that the disease may be brought under control in a given area in the shortest possible time. The following are the main points:

(1) A complete survey of the leprosy infected area is required. The data should include the ages of the patients and the types of cases.

(2) Open cases should be isolated. This should be either in an institution-self-supporting —or in special huts in the patients' own villages, a measure that the villagers should be encouraged to carry out.

(3) Active cases should be treated. Particular attention in this respect should be paid to children who present signs of activity.

(4) Contacts and non-active cases should be followed up and observed periodically.

(5) Propaganda should be carried on, emphasising : (a) That leprosy is an ordinary disease communicated by close contact, and neither venereal nor a curse of God; (b) that only certain stages are infective ; (c) that not every case needs treatment, there being forms which only need periodical watching ; $(d)$ that of the cases that need treatment those which have it early have the best chances of recovery ; $(e)$ that only those cases which are dangerous to the public, need be segregated; others can be treated as out-patients at special clinics or ordinary hospitals; $(f)$ that in early and non-infective stages children need not leave their schools or workers give up their employment, though they should be examined periodically ; $(g)$ that all who have come into close contact with a patient should have periodic examinations; $(h)$ that a healthy body is the best defence for the individual, and the development of a public health sense is one of the greatest factors against the spread of the disease.

In interpreting survey data it is to be realised that a 
high gross incidence is not necessarily an indication of the activity of the disease or of the difficulty of controlling it. Besides the total figures there is needed information as to the proportion of the revealed cases which pass on to the more active stages, and what proportion are of the abortive types. If the cases in a given region are chiefly neural and children do not seem to be infected, then it seems legitimate to conclude that the disease is not on the increase in that area, and that it may be expected to tend gradually to die out without any special measures being taken. If, on the other hand, infective cases are common and the incidence among children high, active measures should be taken to prevent further spread.

I venture to suggest that much of our propaganda is on a wrong basis. We often survey an area with a fanfare of trumpets that arouses interest in the matter, establish outpatient centres without due regard to the conditions that exist, cause the local institutions to be besieged with cases to overflowing, and then rest content that we are dealing with the leprosy problem. The fact often is, I feel certain, that out-patient clinics become silted up with cases which are unsuitable for treatment. These fall into four categories : (1) early abortive cases which need no treatment ; (2) arrested cases without deformity but with permanent areas of hypopigmentation ; (3) deformed arrested (" burntout ") cases ; (4) advanced skin cases which are dangerous to the community and cannot hope to be rendered noninfective within a reasonable time.

One of the reasons why it is impossible to generalise with regard to leprosy prevention is that its prevalence not only varies greatly in different countries, but different districts in the same country show enormous variations. Leprosy seems always to exist in foci, but the factors that cause these foci are imperfectly understood. The main factors that affect the spread of the disease in a given area are : (1) introduction of an infective case into a village previously healthy ; (2) famine or disease lowering the resistance in a village already mildly affected, thus aggravating the actual cases and facilitating further spread; (3) unfavourable climatic and dietetic factors; (4) overcrowding and unhygienic surroundings; (5) industrialisation. However, this is by no means the whole story, and it is just here that patient field work should be repaid. If every detail such as the climate, history, habits, disease, housing, etc., were studied in contrasting districts, both where leprosy is prevalent and where it is not common, certain common 
factors might be elicited and further epidomiological facts ascertained.

The view point with regard to epidemiology and prevention here presented, one submits, simplifies the whole problem. The apparently gigantic task that the health authorities face where there is a great deal of leprosy would be much lessened if it were realised $(a)$ that certain areas might not need special measures for leprosy control, and (b) that if the cases revealed by a survey were properly analysed only a certain proportion of them would need to be treated. With respect to the second point, it has been said that there is in it a fundamental fallacy, namely, that it is impossible in any disease to say whether or not a given case will prove to be abortive without treatment, and as an example in support of this contention tuberculosis has been cited. However, the circumstances in the two diseases are so different that this is not valid. The earliest or slightest lesions in tuberculosis cannot be detected positively and watched. The evidences which many people infected with leprosy exhibit are so early or slight and so inactive, that they. are fairly comparable as regards stage with the undetectable focus of tuberculosis in, say, a single bronchial lymph node, but they are detectable and can be watched. And I maintain that, as many people with such tuberculous foci never develop clinical tuberculosis, so many with analogous foci of leprotic infection do not develop active leprosy. To make due allowance for such cases where they occur in appreciable numbers will lighten in a corresponding degree the task of leprosy control.

It is regretted that the nature of my work has made it impossible to investigate in detail this most interesting aspect of the leprosy problem, and will not permit pursuing it further. It is hoped that those better situated to do so will investigate the matter in a way not yet done. However, one is confident that an evaluation of the abortive case will lead to more accurate epidemiological knowledge and to a revision of general policies of control and prevention of the disease.

\section{REFERENCES.}

(1) Lampe, P. H. J.- " The Organisation of Anti-Leprosy Measures in Surinam." Lep. Rev. 3 (1932) 147-158.

(2) Lampe, P. H. J.- " Has Leprosy a Tendency to Spontaneous Arrest Without Defect?" Lep. Rev. 4 (1933) 23-28.

(3) Chiyuto, S.- " Early Leprotic Changes in Children and their Bearing on the Transmission and Evolution of the Disease." Abst. in : Trop. Dis. Bull. 31 (1934) 5.

(4) Report of the All-India Conference, Calcutta, March, 1933. Lop. in India 5 (1933) No. 3, July. 